Article

\title{
Label-Free, Single Molecule Resonant Cavity Detection: A Double-Blind Experimental Study
}

\author{
Maria V. Chistiakova ${ }^{\dagger}, \mathrm{Ce} \mathrm{Shi}^{\dagger}$ and Andrea M. Armani * \\ Mork Family Department of Chemical Engineering and Materials Science, \\ University of Southern California, 3651 Watt Way, Los Angeles, CA 90089, USA; \\ E-Mails: chistiak@usc.edu (M.V.C.); cshi@usc.edu (C.S.) \\ $\dagger$ These authors contributed equally to this work. \\ * Author to whom correspondence should be addressed; E-Mail: armani@usc.edu; \\ Tel.: +1-213-740-4428; Fax: +1-213-740-7797. \\ Academic Editor: M. Selim Ünlü
}

Received: 11 November 2014 / Accepted: 5 March 2015 / Published: 16 March 2015

\begin{abstract}
Optical resonant cavity sensors are gaining increasing interest as a potential diagnostic method for a range of applications, including medical prognostics and environmental monitoring. However, the majority of detection demonstrations to date have involved identifying a "known" analyte, and the more rigorous double-blind experiment, in which the experimenter must identify unknown solutions, has yet to be performed. This scenario is more representative of a real-world situation. Therefore, before these devices can truly transition, it is necessary to demonstrate this level of robustness. By combining a recently developed surface chemistry with integrated silica optical sensors, we have performed a double-blind experiment to identify four unknown solutions. The four unknown solutions represented a subset or complete set of four known solutions; as such, there were 256 possible combinations. Based on the single molecule detection signal, we correctly identified all solutions. In addition, as part of this work, we developed noise reduction algorithms.
\end{abstract}

Keywords: biosensors; optical resonators; single molecule detection 


\section{Introduction}

Over the past several decades, optical resonant cavities have demonstrated the ability to perform biological and chemical detection experiments for a wide range of applications [1-37]. While the initial focus was on spherical whispering gallery mode cavities, such as microdroplets [38,39] and silica microspheres $[13,40]$, as lithography techniques became more broadly accessible, the variety of shapes and materials quickly expanded to include rings [12,41], toroids [10,42,43], photonic crystals $[15,26,28,35]$, and many others. Additionally, as interest in pushing the detection sensitivity to the single molecule level has increased, the method of performing detection has experienced a rapid growth. While the initial demonstrations relied on detecting a refractive index change or fluorescence, more recent experiments involved detecting a change in the optical loss of the system [5], a phase change [44], back-scatter [6], or optical mode splitting [10,11]. However, the index change approach still remains the most straightforward method as it involves the minimum amount of equipment.

One reason that whispering gallery mode optical cavities are ideally suited for refractometric detection is that the optical field is confined in a circulating orbit, allowing it to sample the surface multiple times [45]. The number of orbits is directly related to the quality factor, or Q, of the cavity. It was this repeated interaction which first inspired researchers to develop these devices into sensors. Namely, the sampling results in an amplification of the signal through several distinct mechanisms: (1) every interaction acts as a unique detection event (detection is proportional to the number of circulations, which is proportional to Q); (2) the detection signal is proportional to the interaction strength of the particle and the optical field (detection is related to the intensity of the optical field at the surface); and (3) detection is performed by tracking the linewidth (detection is proportional to Q). Therefore, even a simple, intuitive analysis of this system leads one to determine that geometry and Q play critical roles in the overall sensing signals.

Given the increased interest in using these devices in diagnostics applications [1], we have performed a double-blind experiment at the single molecule level using silica optical resonant cavities. This type of experiment is standard practice in the pharmaceutical industry (also called double-blind, placebo-controlled) $[46,47]$ and is widely recognized as the most rigorous approach to validate the reliability of a sensor's response because it removes the experimenter's bias. However, to our knowledge, such a study has yet to be performed with ultra-high-Q cavities at the single molecule level to date. Namely, unlike previous work in the field of resonant cavity sensing, which injected known solutions and demonstrated detection of those known analytes, we have injected unknown solutions, and successfully identified their contents based solely on the generated signal.

\section{Experimental Section}

Double-blind experiments are the classic method for verifying a diagnostic device's ability to perform accurate identification of a chemical or biological species. In this type of experiment, a series of solutions will be prepared by one researcher (e.g., a, b, c). This series will be given to a second person who will mix and re-name the solutions (e.g., 1, 2, 3). They will also create a key that will be placed in a sealed envelope until the end of the experiment (e.g., $1=c, 2=a, 3=b)$. The re-named set of solutions will be given to the researchers who are performing the experiment. Once the experiment 
is over, the researchers email both the first and second set of researchers their guess as to the identity of the solutions. At that point, the second person reveals the answers.

There are several variations on this general paradigm, depending on the complexity or difficulty desired. For example, the researchers could be told that each solution is unique. Alternatively, they could be given a long list of solutions and told that their solutions are a subset. Depending on the amount of information provided to the experimenter, the probability of arriving at the correct answer rapidly diminishes.

\subsection{Double-Blind Strategy}

In the present series of detection experiments, we are given a total of eight solutions: (1) four known solutions and (2) four unknown solutions. The four known solutions provide a ruler and are labeled with their contents: phosphate buffered saline buffer (PBS, $10 \mathrm{mM}, \mathrm{pH}$ 7.0, Sigma Aldrich), bovine serum albumin (BSA, $66 \mathrm{kDa}$, Sigma Aldrich), Streptavidin (SA, $60 \mathrm{kDa}$, Sigma Aldrich), and Streptavidin-labelled polystyrene beads (SA-PS beads, $100 \mathrm{~nm}$ pre-functionalization, Sigma Aldrich). The second set of four solutions is the unknown samples. All reagents (PBS) needed to create the dilutions are also supplied. It should be noted that only after the double-blind process is completed are we told the material molecular weight and manufacturer. This approach removes any bias. We are told that the unknown set of solutions represent either a subset or complete set of the known solutions. Based on this paradigm, there are 256 possible combinations. Therefore, it is highly unlikely that the correct answer would be arrived at by chance.

Our approach for identifying the different solutions is outlined in Figure 1. Specifically, it is straightforward to determine if a solution contained buffer or SA-PS beads. Determining if a solution contained BSA or SA required more finesse, as these molecules are approximately the same molecular weight. Therefore, we had to employ a surface functionalization capable of discriminating between BSA and SA. We chose biotin for two reasons: (1) it would improve the reliability of the SA-PS bead detection and (2) it is extremely robust, thus removing environmental instability as a potentially confounding variable.

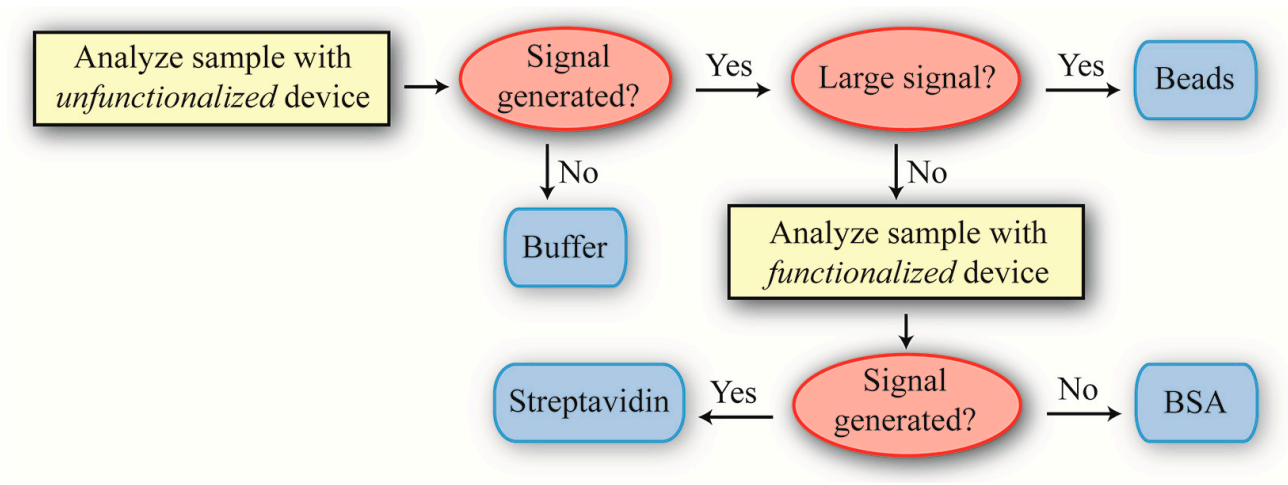

Figure 1. The decision tree used for differentiating between the four samples. Depending on the result from the first experiment using unfunctionalized devices, a second experiment using biotin-functionalized devices might be performed to further analyze a sample's contents. This hierarchical approach of prioritizing decision-making is commonly used in Artificial Intelligence. 


\subsection{Solution Preparation}

All solutions are provided to the Armani Lab by an independent observer. The four known solutions (PBS buffer, BSA, SA, and SA-PS beads) are at a concentration of $1 \mathrm{nM}$. Extra PBS buffer is also given to the Armani Lab to allow dilutions of the four unknown solutions to be made. The remaining four solutions are labeled Dog, Cat, Pig, and Cow, and are also at $1 \mathrm{nM}$.

A series of solutions for detection experiments are made using serial dilution to $10 \mathrm{aM}, 500 \mathrm{aM}, 10 \mathrm{fM}$, and $10 \mathrm{pM}$ using the provided PBS buffer. Based on previous work, we focused on the $500 \mathrm{aM}$ and $1 \mathrm{fM}$ solutions, and the results from the $1 \mathrm{fM}$ measurements are presented here. The results from the $10 \mathrm{pM}$ experiments were also used to cross-verify the results for the Cat solution. For that solution, we were concerned that the protein might have precipitated out of the PBS, resulting in an incorrect initial or starting concentration. Solutions were stored in the refrigerator until the experiments, at which point they were allowed to warm up to room temperature before testing.

\subsection{Device Fabrication and Functionalization}

Two different cavities are used in the present work: microspheres and microtoroids (Figure 2a,b). The toroidal cavities are fabricated using the standard three-step method, which is summarized as follows $[48,49]$. First, circular oxide pads are lithographically defined on a silicon wafer. Then, oxide microdisks are undercut using xenon difluoride. Finally, toroidal microcavities are formed using a carbon dioxide reflow process. Final device diameters ranged from 95 to $105 \mu \mathrm{m}$. The silica microspheres are fabricated from optical fiber by first removing the protective polymer layer from standard optical fiber. Next, it is reflowed in a carbon dioxide laser. Final device diameters ranged from 180 to $200 \mu \mathrm{m}$. These device diameters are chosen to minimize the radiation losses, which can occur if the device size shrinks below a critical radius [50-52].
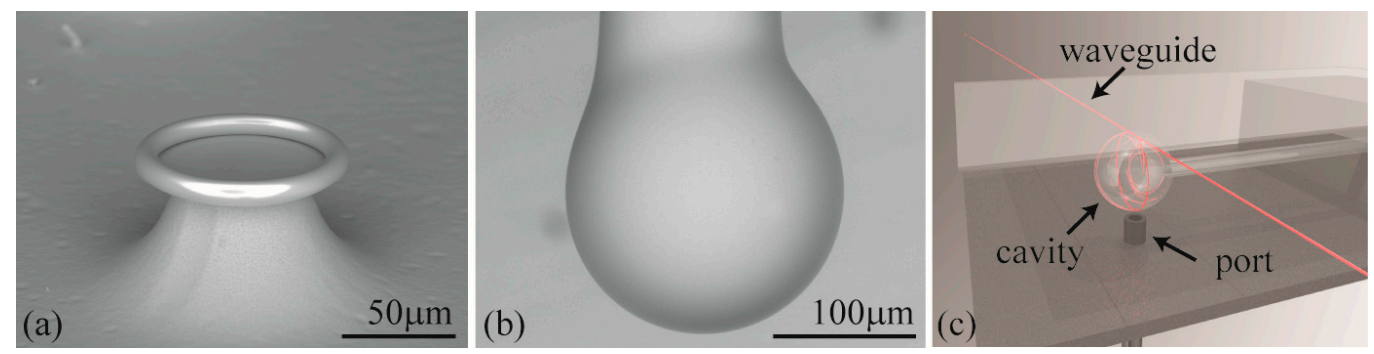

Figure 2. Scanning electron microscope image of a (a) toroid and a (b) microsphere resonator; (c) Rendering of the testing set-up with key components indicated. The liquid chamber consists of a steel holder and a glass cover slip. If a toroid is used in the experiments, then the injection port is located on the side.

To functionalize the surface of the devices with biotin, we use a previously developed NHS-Ester chemistry method [53]. In this method, the silica surface is first hydroxylated using an oxygen plasma treatment. Then, the surface is aminated using chemical vapor deposition of 3-aminopropyltrimethoxysilane. Finally, the devices are incubated in a solution of N-hydroxysuccinimido-biotin in dimethylsulfoxide. This process forms stable amide bonds and a uniform layer of biotin on the device surface. However, 
there are numerous other surface chemistry methods which have been developed and which could have been used to perform these experiments [54].

\subsection{Device Characterization}

Before performing a detection experiment, the quality factor of a device is determined by measuring the resonant linewidth or full-width-half-max (FWHM) of the cavity in buffer. A tunable laser (Newport, Velocity series) is coupled into a tapered optical fiber, which is brought adjacent to the resonant cavity using a nanopositioning stage. The laser is scanned across the resonance, and the spectral resolution is determined by the scan range divided by the number of data points acquired by the oscilloscope over that range. For the present series of experiments, the total wavelength scan ranges from $1 \mathrm{~nm}$ to approximately $0.05 \mathrm{~nm}$, and the PCI card acquires 300,000 data points evenly spaced over this range. Therefore, the spectral resolution ranges from of $3.334 \mathrm{fm}$ to approximately $0.166 \mathrm{fm}$. The evanescent optical field from the taper is coupled into the cavity, and the transmitted power is monitored and recorded on a NI PCI high-speed digitizer/oscilloscope with a temporal resolution of $250 \mathrm{MegaSamples/sec} \mathrm{real-time.}$

Both the fiber and the cavity are sandwiched between the sample stage and a coverslip, and the chamber is filled with approximately $350 \mu \mathrm{L}$ of buffer (Figure $2 \mathrm{c}$ ). The spectra is recorded in the under-coupled regime, allowing an accurate measurement of the cavity intrinsic Q [55]. All toroid measurements are performed at $633 \mathrm{~nm}$, and all microsphere measurements are performed at $765 \mathrm{~nm}$. All non-functionalized cavities have intrinsic Q factors above 10 million at the start of the experiments.

\subsection{Sensing Experiments Procedure}

The coupling is increased for the detection measurements. The output power during the detection measurements is greater than $1 \mathrm{~mW}(633 \mathrm{~nm})$ or $2.3 \mathrm{~mW}(765 \mathrm{~nm})$. At least $90 \%$ of the laser power is coupled into the cavity. It is important to note that at these circulating powers, nonlinear phenomena such as Raman lasing [56] and opto-thermal broadening [5] have been previously observed in these devices operating under these conditions (same size device at the same wavelength in buffer).

To begin a detection measurement, the background signal is measured to establish the noise level. The minimum position and transmission error, the resonance is recorded for approximately $200 \mathrm{~s} \mathrm{at} 10 \mathrm{~Hz}$ (10 points per second). After recording two noise measurements, the detection measurements are started. The LabView program begins recording the position of the peak just before the solution of interest is injected. The flow rate of each solution is set to be $50 \mu \mathrm{L} / \mathrm{min}$. The solution is injected for two min, for a total injected volume of $100 \mu \mathrm{L}$, and then the flow is stopped. During this time, 2000 data points are recorded. Then, the liquid chamber is flushed with excess buffer, and the experiment is repeated. We chose to flow the solutions across the device surface instead of relying purely on diffusion as it this approach allows a more controlled delivery of the material to the sensing device surface [57].

The results are partially analyzed immediately after the measurement to provide instruction according to the design tree outlined in Figure 1 as to the next experiment. After the unknown solutions are tested, the known solutions are used as a reference, assuming the device is still operational; namely the Q and the circulating power are not degraded and the taper is not broken. 


\subsection{Data Analysis Methods}

The two common approaches for performing resonant cavity detection are: (1) tracking the resonant cavity wavelength ( $x$-axis position) and (2) tracking the transmission or coupling ( $y$-axis position). It is extremely difficult to track both in parallel using the standard resonant cavity-testing set-up, which relies on an external oscilloscope (wavelength tracking) or a power meter (transmission). However, when acquiring data at high acquisition speeds and high input powers, changes in coupling can give rise to small wavelength changes, leading to a high error rate or background noise. To solve this problem, we replaced the external oscilloscope with an integrated PCI high-speed digitizer/oscilloscope card. Using this integrated system in combination with a custom LabView data acquisition code, we are able to record both the wavelength and the transmission simultaneously. We can then apply a post-processing filter and remove all wavelength shift data where the transmission fluctuated by more than a predetermined amount. An image graphically explaining this analysis process is shown in Figure 3.

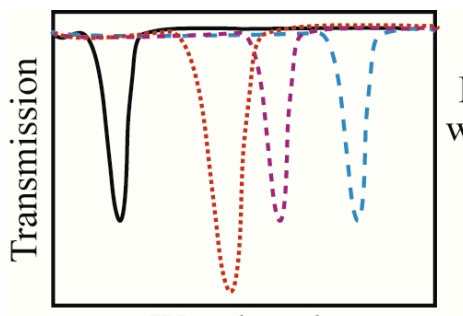

(a) Wavelength

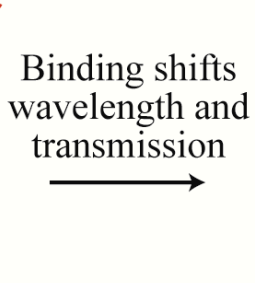

(b)

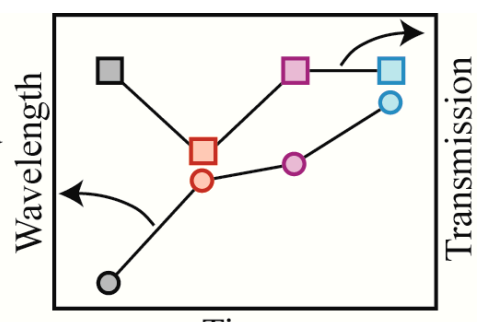

Time

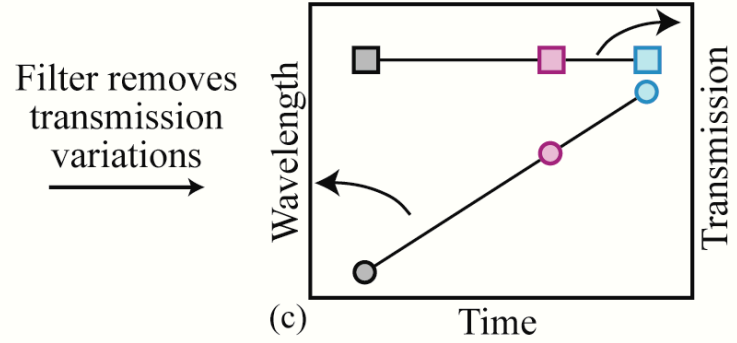

Figure 3. Image of the data analysis path. (a) The resonant wavelength red-shifts as proteins bind on the surface. Additionally, the transmission changes due to coupling instabilities; (b) if the four results in part (a) are re-plotted on a dual y-axis graph, the relationship between the wavelength and the transmission becomes apparent. Additionally, it becomes clear that this relationship can convolute the data analysis; (c) by applying a filter to remove coupling variations, the relationship between wavelength and time becomes evident.

Once the variation in coupling is removed from the data, the results are further analyzed by plotting the normalized resonant wavelength shift $(\Delta \lambda)$, or $\lambda_{\mathrm{i}}-\lambda_{0}$, where $\lambda_{\mathrm{i}}$ is the $i$ th resonant wavelength and $\lambda_{0}$ is the initial resonant wavelength value. From these results, the instantaneous shift $(\delta \lambda)$ is determined $\left(\lambda_{\mathrm{i}}-\lambda_{\mathrm{i}-1}\right)$ and plotted as a histogram. However, this histogram includes instantaneous shifts for both the binding events and the noise. Therefore, this analysis is performed for both the detection experiments and for the control noise measurements. The histogram of the noise is fit to a Gaussian, and the $3 \sigma$ value is determined. This decision represents our focus on optimizing the device and data analysis for minimum false-positives. However, it also means that some of our binding events will be excluded 
from analysis because they will fall within the $3 \sigma$ value. Finally, the instantaneous shift from the noise is removed from the detection results, leaving only the signal from the protein binding.

\section{Results and Discussion}

\subsection{Device Selection}

It is well-known that the resonant shift is dependent on the refractive index contrast and the mode volume. Recently, it was also demonstrated that the evanescent field extension into the environment plays a significant role. In the present series of measurements using two different device geometries, we also observe consistency issues, both in the resonant frequency shift due to binding, as well as the background noise due to thermal effects. For example, in toroidal cavities, it is possible to achieve an athermal condition by balancing the positive thermo-optic coefficient of silica and the negative thermo-optic coefficient of water. However, due to the difference in optical mode profile and overlap with the environment, this condition is not possible with spheres. This change gives rise to a completely different background noise profile and thermal drift behavior.

Therefore, while the toroidal data or sphere data is self-consistent, comparing between device geometries is problematic. Investigations are currently underway to elucidate all of the different contributors, both geometry-specific parameters as well as others. However, at this time, it is not appropriate to directly compare results between devices given the previous work in the field, which has shown these numerous geometry-specific dependencies. As such, to facilitate the discussion of the results, we are focusing on the toroid data for the present work.

\subsection{Wavelength Shift and Background Noise}

At the start of every experiment, a background noise measurement is performed, and representative instantaneous noise histograms are shown in Figure 4. It is important to note that researchers in this field frequently interchange the names instantaneous shift $(\delta \lambda)$ and the normalized shift $(\Delta \lambda)$ with the term resonant wavelength shift. However, as can be seen in Figure 4a,b, these are distinct values and represent the results from different calculations. The results in Figure $4 \mathrm{~b}$ can be more clearly understood if plotted as a histogram, where the $y$-axis represents the number of times that a single $\delta \lambda$ value occurs (Figure 4c). As expected from a noise data set, it is very symmetric and exhibits a Gaussian distribution around the $\delta \lambda=0$ point. For all sensing measurements, we set the noise threshold at 3 standard deviations $(3 \sigma)$ of the mean of this histogram. $3 \sigma$ accounts for $99.7 \%$ of the noise. This experimentally-derived value is used to calculate the signal:noise (SNR) for each detection result. This thresholding approach works well, assuming a Gaussian noise distribution.

However, because detection measurements can be performed either during the day or at night, it is useful to compare the noise during these two times. The data in Figure $4 \mathrm{a}-\mathrm{c}$ are taken during the day whereas the data in Figure 4d are taken at night. 

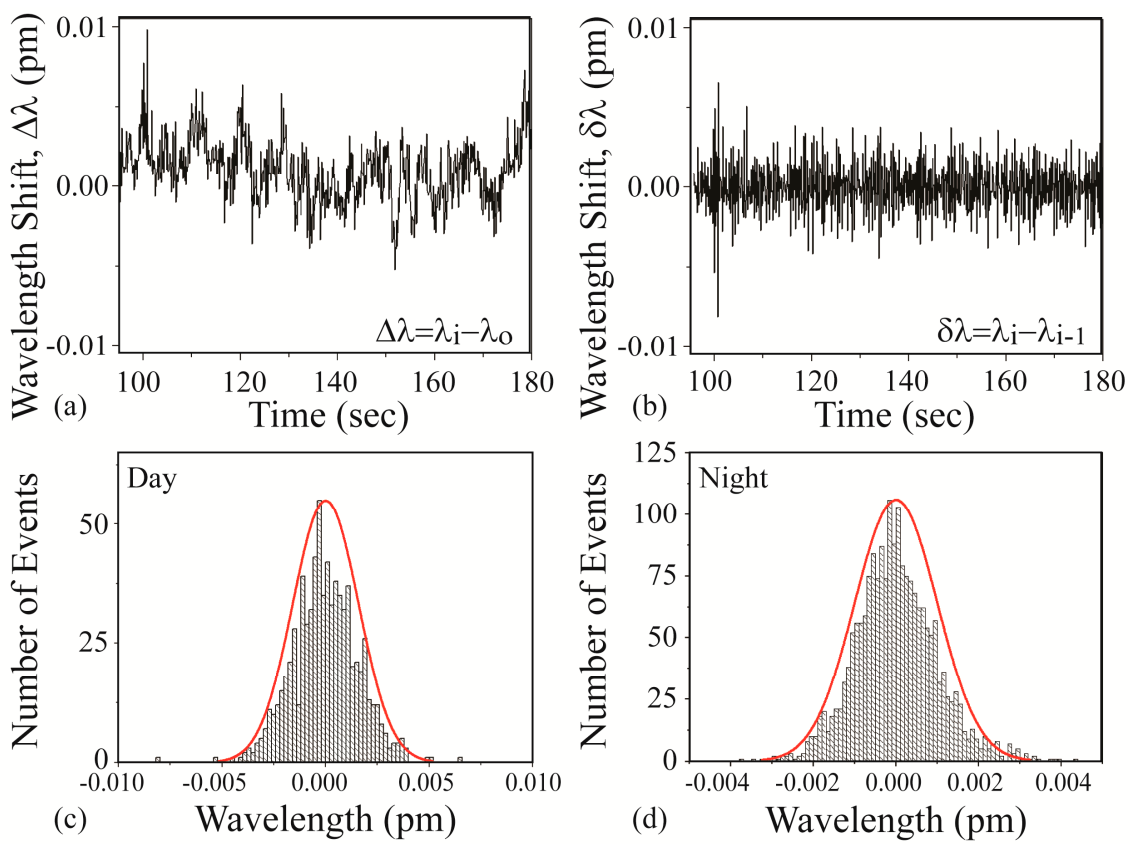

Figure 4. Noise measurements. (a) Normalized resonant wavelength shift $(\Delta \lambda)$ measured during the day; (b) instantaneous resonant wavelength shift $(\delta \lambda)$ of part (a); (c) histogram of the values in part (b); (d) histogram of values of an instantaneous resonant wavelength shift $(\delta \lambda)$ measured at night.

As can be observed, the noise during the day is significantly higher (nearly twice) than the noise in the evening. Given this dependence, the noise is measured for each experiment uniquely. While this noise could be attributed to a range of noise sources, given the reproducibility of the data and the uniformity of the noise, we believe it is due to the air conditioning, which is reduced in the evening due to California energy saving regulations. At the time of these experiments, both lasers and the accompanying detectors were under one year old; therefore, noise due to the laser or the grating degradation was minimal. These findings inspired several subsequent investigations into the noise limitations in these types of experiments and experimental set-ups and alternative methods of analyzing the detection signal to circumvent these limitations $[44,58,59]$.

\subsection{Detection Measurements}

Representative detection results and the resulting histograms from all four unknown solutions are shown in Figures 5-8. Each measurement is shown with the noise data set, and the results will be discussed individually.

\subsubsection{Cat Solution}

Figure $5 \mathrm{a}-\mathrm{c}$ is the background noise measurements. Based on a fit to the histogram shown in Figure $5 \mathrm{c}$, it was determined that the $3 \sigma$ noise is $0.00502 \mathrm{pm}$. This value is plotted as dashed lines on Figure $5 \mathrm{~b}$,e. Figure $5 \mathrm{~d}-\mathrm{f}$ is the detection results using an unfunctionalized cavity upon the introduction of the Cat solution. During these measurements, the Q and the input power did not change. 
It is important to note that the background noise was not removed in these measurements, as the magnitude of the noise-based shift is similar to that of solution-induced shift. This relationship is clearly observed by comparing Figure 5c,f or looking at the dashed lines in Figure 5e.

As a result, the SNR is approximately 1 . An SNR of 1 is conventionally defined in the radar community as an undetectable signal. In the present work, that would translate to a "blank" solution, or the buffer. Therefore, based on the previously defined decision tree (Figure 1), the Cat solution is buffer.
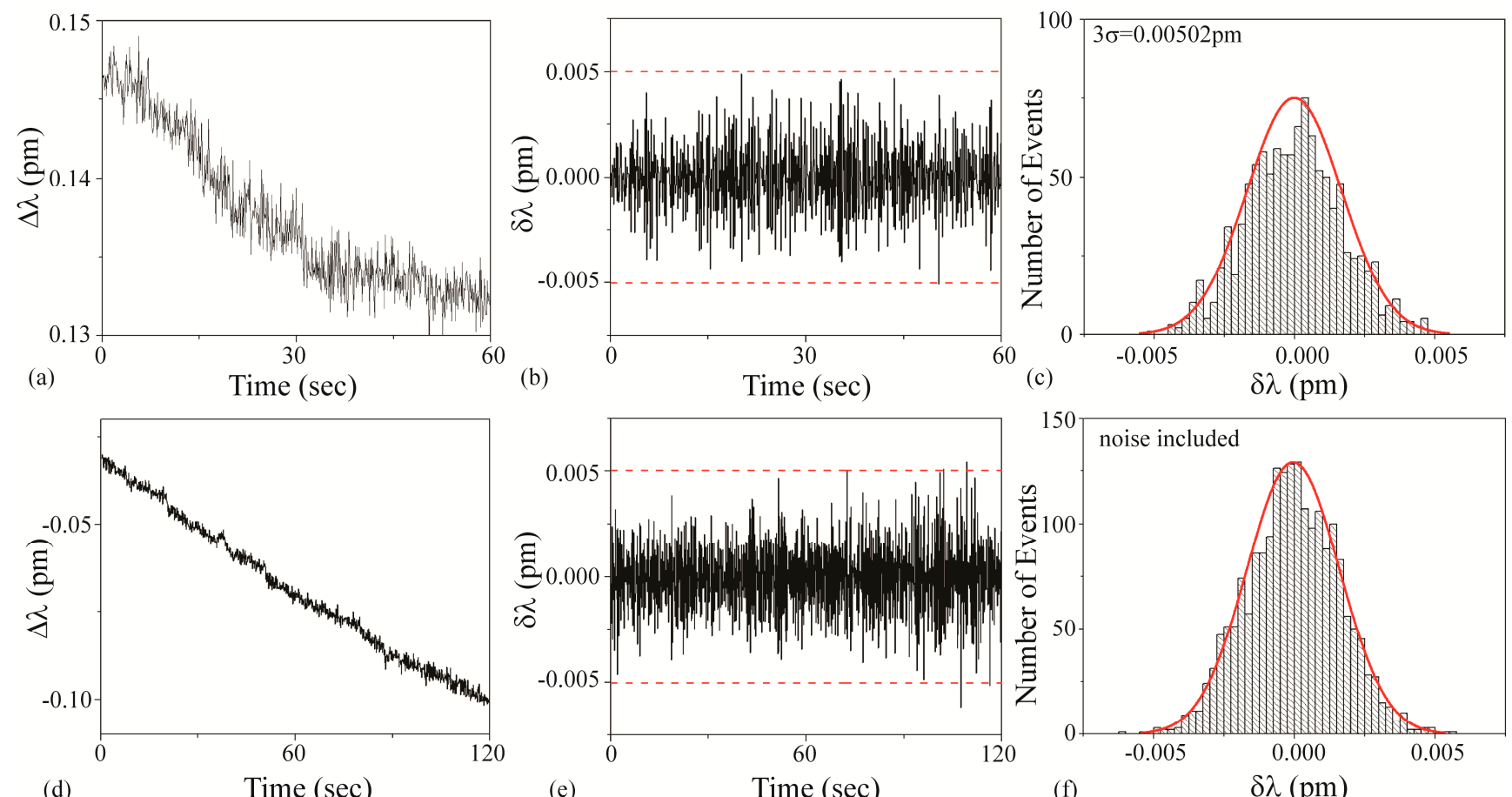

(e)

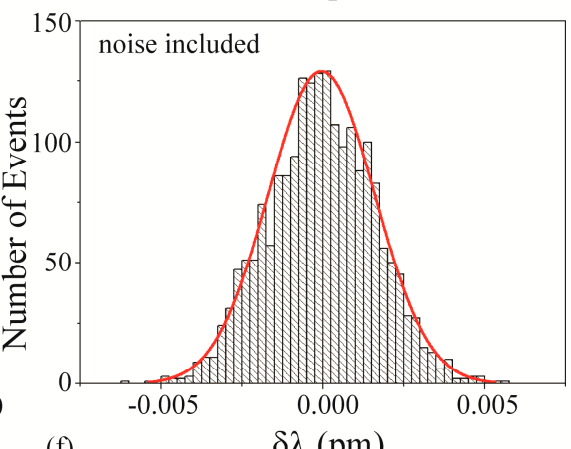

Figure 5. Cat solution: (a-c) background and (d-f) detection experiments. (a) Background wavelength shift $(\Delta \lambda)$ results; (b) background instantaneous wavelength shift $(\delta \lambda)$ results calculated from part (a) data. The dashed line is the $3 \sigma$ noise level determined in part (c); (c) histogram of background $\delta \lambda$ results calculated from part (b) data. Based on these results, the $3 \sigma$ noise is $0.00502 \mathrm{pm}$; (d) wavelength shift $(\Delta \lambda)$ upon injection of Cat solution; (e) instantaneous wavelength shift $(\delta \lambda)$ results calculated from part (d) data. The dashed line is the $3 \sigma$ noise level determined from the histogram shown in part (c); (f) histogram of $\delta \lambda$ results shown in part (e). The background noise has not been subtracted from the histogram.

\subsubsection{Cow Solution}

Figure $6 \mathrm{a}-\mathrm{c}$ is the background noise measurements. Based on a fit to the histogram shown in Figure $6 \mathrm{c}$, it was determined that the $3 \sigma$ noise is $0.00429 \mathrm{pm}$. This value is plotted as dashed lines on Figure $6 \mathrm{~b}, \mathrm{~d}$ inset, and $6 \mathrm{e}$ inset. Figure $6 \mathrm{~d}-\mathrm{f}$ are the detection results using an unfunctionalized cavity upon the introduction of the Cow solution. During the injection, the $\mathrm{Q}$ and the transmitted power steadily decreased, most likely due to the agglomeration of the microparticles on the taper and the microcavity. Additionally, an increase in scattering at the surface of the cavity was observed during the experiments with a top view optical microscope and camera. This power and Q decrease limited the duration of experiments using these solutions. 
As can be observed in Figure 6, the results from the Cow solution demonstrate two distinctly different shifts: a small shift $(\sim 0.007 \mathrm{pm})$ and a large shift $(\sim 0.2 \mathrm{pm})$. We concluded that the small shift is due to free streptavidin in the solution and the large shift is due to the SA-functionalized PS bead complex. This bi-magnitude shift was un-intended in the initial experimental design; however, it did reveal the ability of the method to detect impurities in a solution. This effect was further studied in subsequent work, looking into both binding rates and mass transport related effects [60]. Because of the dual signal, two different signal:noise calculations should be performed. Based on the raw data, for the large bead-streptavidin complex, the SNR is $\sim 47$ and for the streptavidin, the SNR is $\sim 2$.

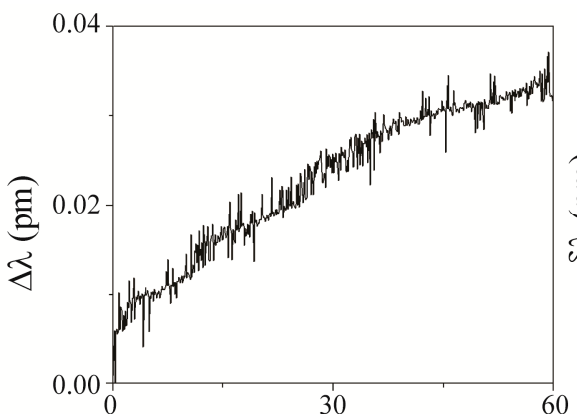

(a)

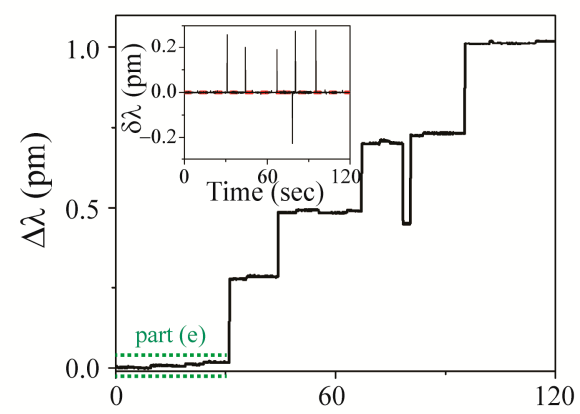

(d)

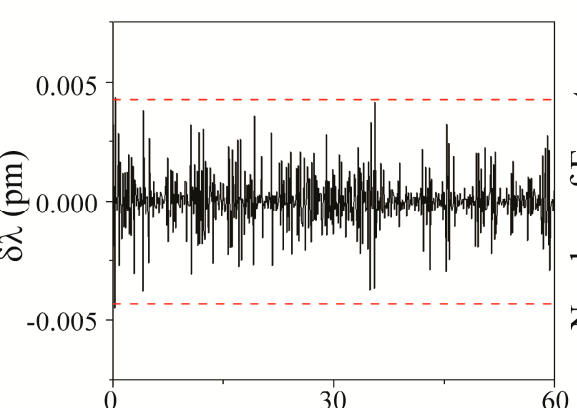

(b)

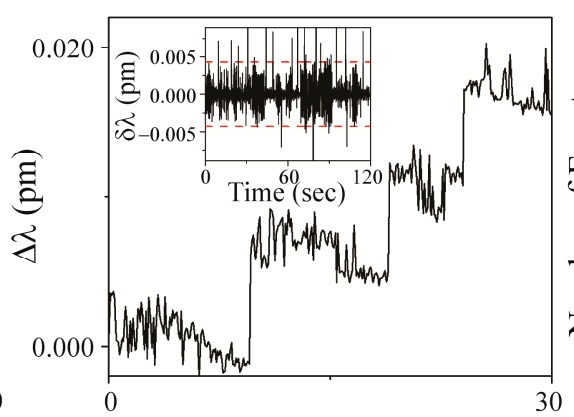

(e)

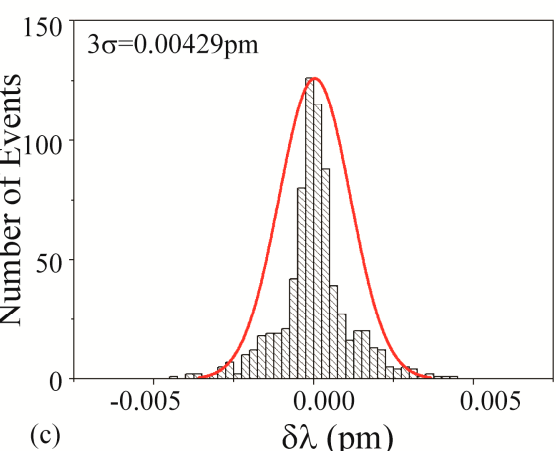

(c) $\delta \lambda(\mathrm{pm})$

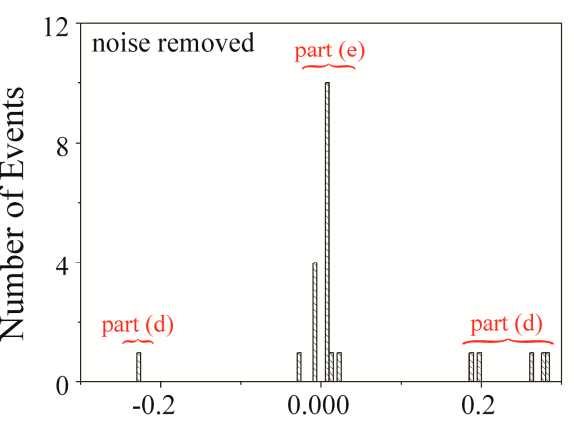

(f) $\delta \lambda(\mathrm{pm})$

Figure 6. Cow solution: $(\mathbf{a}-\mathbf{c})$ background and $(\mathbf{d}-\mathbf{f})$ detection experiments. (a) Background wavelength shift $(\Delta \lambda)$ results; (b) background instantaneous wavelength shift $(\delta \lambda)$ results calculated from part (a) data. The dashed line is the $3 \sigma$ noise level determined in part (c); (c) histogram of background $\delta \lambda$ results calculated from part (b) data. Based on these results, the $3 \sigma$ noise is $0.00429 \mathrm{pm}$; (d) wavelength shift $(\Delta \lambda)$ upon injection of Cow solution. Inset: Instantaneous wavelength shift $(\delta \lambda)$ results calculated from this data. The dashed line is the $3 \sigma$ noise level determined from the histogram shown in part (c); (e) wavelength shift $(\Delta \lambda)$ during first $30 \mathrm{~s}$ upon injection of Cow solution (region indicated in green in part (d)), highlighting small shifts. Inset: Instantaneous wavelength shift $(\delta \lambda)$ results calculated from this data. The dashed line is the $3 \sigma$ noise level determined from the histogram shown in part (c); (f) histogram of $\delta \lambda$ results shown in part (d). The background noise has been subtracted from the histogram. Both the small and the large shifts are evident.

\subsubsection{Dog Solution}

In initial detection experiments characterizing Dog solution with unfunctionalized devices, shifts of approximately $0.01 \mathrm{pm}$ are observed. This magnitude could correspond to either BSA or streptavidin. 
Therefore, to differentiate between BSA and streptavidin, the Dog solution is tested with a biotin-functionalized device. During the injection and detection measurements, the $\mathrm{Q}$ and transmitted power did not change.

Figure $7 \mathrm{a}-\mathrm{c}$ is the background noise measurements using a functionalized device. Based on a fit to the histogram shown in Figure $7 \mathrm{c}$, it was determined that the $3 \sigma$ noise is $0.00552 \mathrm{pm}$. This value is plotted as dashed lines on Figure $7 \mathrm{~b}$,e. Figure $7 \mathrm{~d}-\mathrm{f}$ is the detection results.

As can be observed in Figure 7, the small shifts, which are indicative of small protein binding events, are still present. Based on the decision tree outlined in Figure 1, we conclude that the Dog solution contains streptavidin. Based on the raw data, the SNR is $\sim 2$.
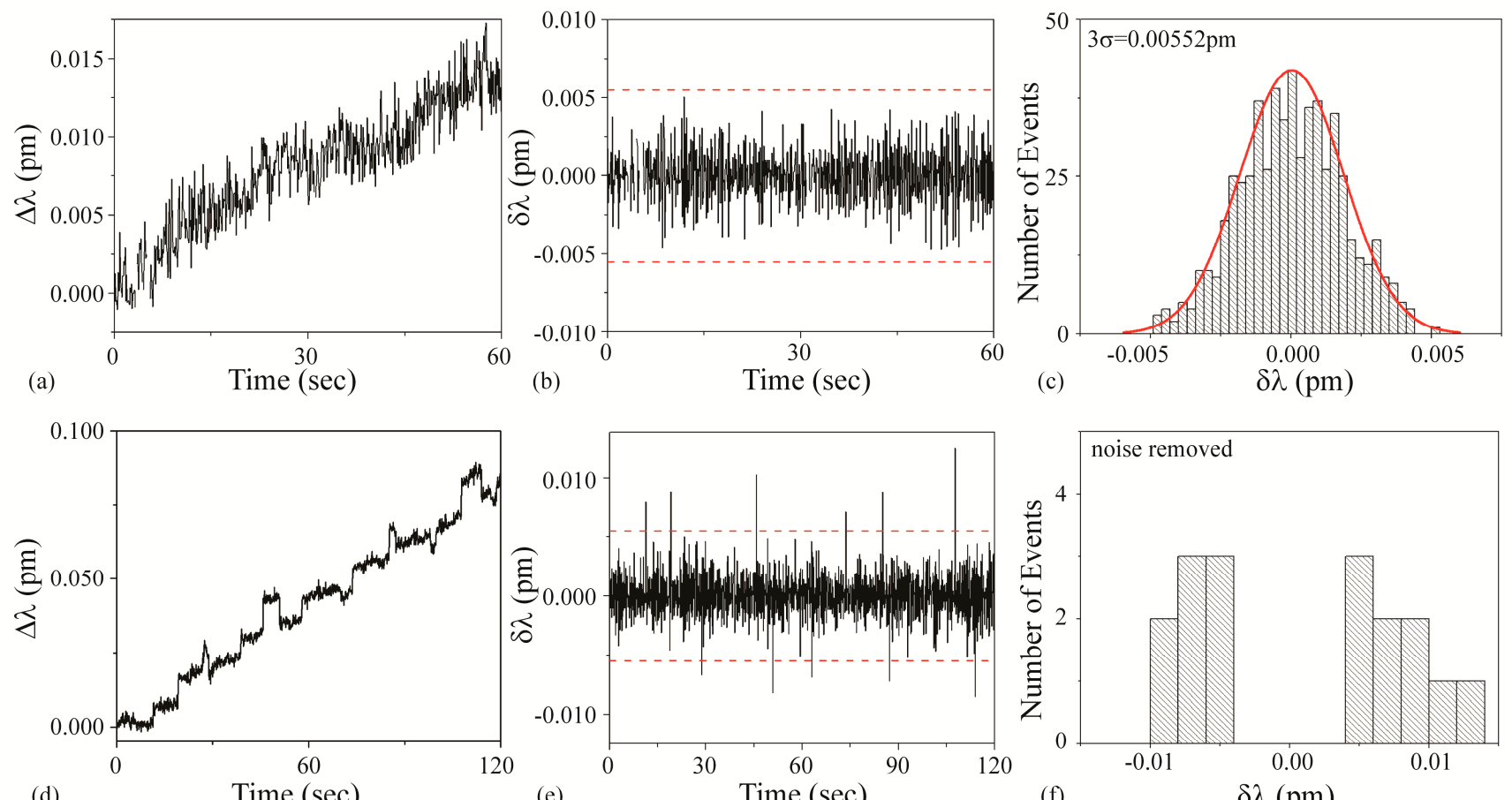

(d)

Time $(\mathrm{sec})$

(e)

(f)

$\delta \lambda(\mathrm{pm})$

Figure 7. Dog solution: (a-c) background and (d-f) detection experiments. (a) Background wavelength shift $(\Delta \lambda)$ results; (b) background instantaneous wavelength shift $(\delta \lambda)$ results calculated from part (a) data. The dashed line is the $3 \sigma$ noise level determined in part (c); (c) histogram of background $\delta \lambda$ results calculated from part (b) data. Based on these results, the $3 \sigma$ noise is $0.00552 \mathrm{pm}$; (d) wavelength shift $(\Delta \lambda)$ upon injection of Dog solution; (e) instantaneous wavelength shift $(\delta \lambda)$ results calculated from part (d) data. The dashed line is the $3 \sigma$ noise level determined from the histogram shown in part (c); (f) histogram of $\delta \lambda$ results shown in part (e). The background noise has been subtracted from the histogram.

\subsubsection{Pig Solution}

Figure $8 \mathrm{a}-\mathrm{c}$ is the background noise measurements. Based on a fit to the histogram shown in Figure $8 \mathrm{c}$, it was determined that the $3 \sigma$ noise is $0.00489 \mathrm{pm}$. This value is plotted as dashed lines on Figure $8 \mathrm{~b}, \mathrm{~d}$ inset, and $8 \mathrm{e}$ inset. Figure $8 \mathrm{~d}-\mathrm{f}$ are the detection results using an unfunctionalized cavity upon the introduction of the Pig solution. During the injection, the Q and the transmitted power steadily decreased and an increase in scattering on the cavity surface were observed. These effects 
were most likely due to the agglomeration of the microparticles on the taper and the microcavity. This power and Q decrease limited the duration of experiments using these solutions.

As can be observed in Figure 8, the results from the Pig solution demonstrate two distinctly different shifts: a small shift $(\sim 0.007 \mathrm{pm})$ and a large shift $(\sim 0.2 \mathrm{pm})$. We concluded that the small shift is due to the presence of free streptavidin in the solution and the large shift is due to the SA-functionalized PS bead complex. This bi-magnitude shift was un-intended in the initial experimental design; however, it did reveal the ability of the method to detect impurities in a solution. Because of the dual signal, two different signal:noise calculations should be performed. Based on the raw data, for the large bead-streptavidin complex, the SNR is $\sim 40$ and for the streptavidin, the SNR is $\sim 1.5$. It is important to note that because the Pig and the Cow solutions are both the PS-bead complex, by default, not all of the known solutions (buffer, BSA, SA, and PS bead complex) will be in the set of unknowns.

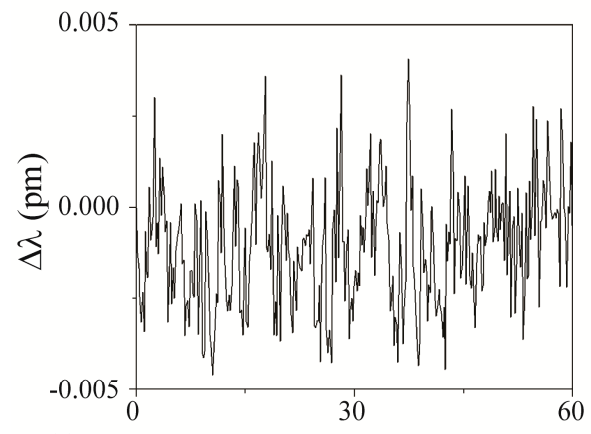

(a)

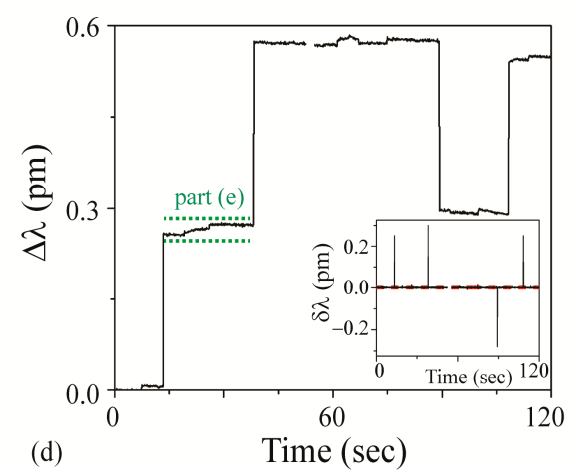

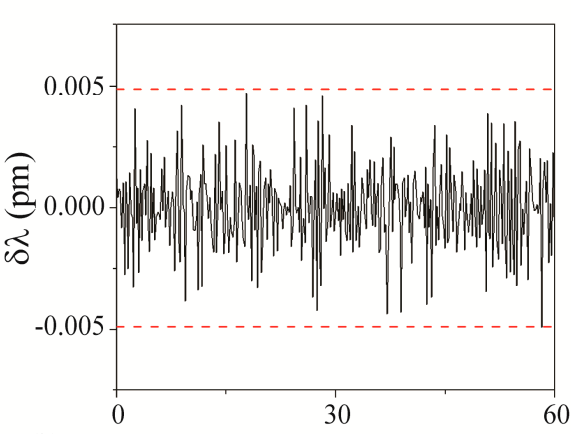

(b)

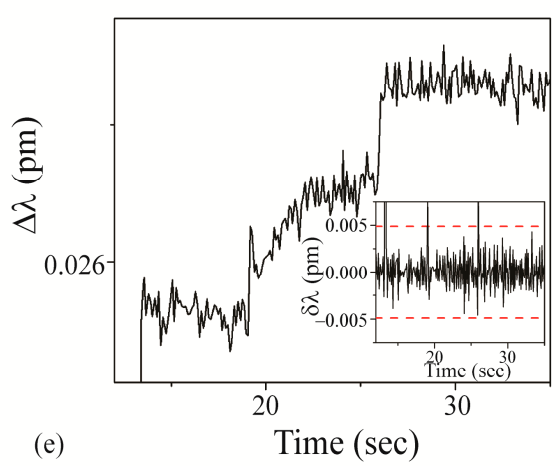

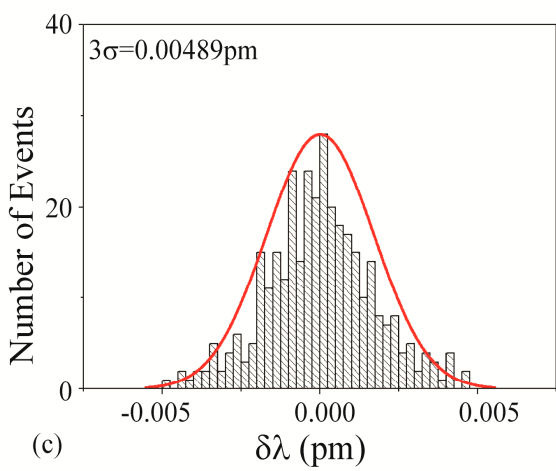

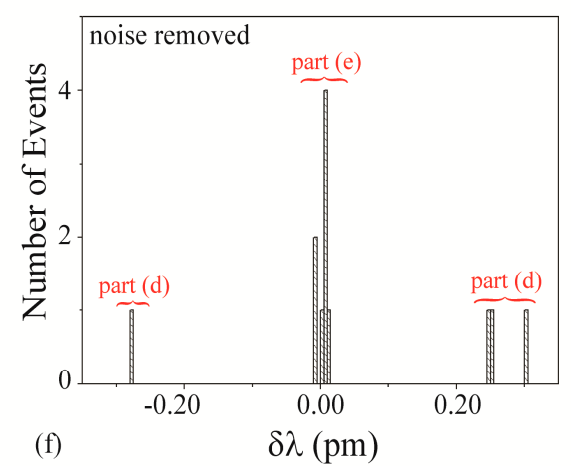

Figure 8. Pig solution: $(\mathbf{a}-\mathbf{c})$ background and $(\mathbf{d}-\mathbf{f})$ detection experiments. (a) Background wavelength shift $(\Delta \lambda)$ results; (b) background instantaneous wavelength shift $(\delta \lambda)$ results calculated from part (a) data. The dashed line is the $3 \sigma$ noise level determined in part (c); (c) Histogram of background $\delta \lambda$ results calculated from part (b) data. Based on these results, the $3 \sigma$ noise is $0.00489 \mathrm{pm}$; (d) wavelength shift $(\Delta \lambda)$ upon injection of Pig solution. Inset: Instantaneous wavelength shift $(\delta \lambda)$ results calculated from this data. The dashed line is the $3 \sigma$ noise level determined from the histogram shown in part (c); (e) wavelength shift $(\Delta \lambda)$ upon injection of Pig solution (region indicated in green in part (d)), highlighting small shifts. Inset: Instantaneous wavelength shift $(\delta \lambda)$ results calculated from this data. The dashed line is the $3 \sigma$ noise level determined from the histogram shown in part (c); (f) histogram of $\delta \lambda$ results shown in part (d). The background noise has been subtracted from the histogram. Both the small and the large shifts are evident. 


\subsection{Summary of Measurements}

Table 1 summarizes all of the results and experimental observations. It also includes the prediction based on the experimental results and the actual solution from the sealed key. It is notable that the experimental results were able to accurately predict all four of the solutions. Based purely on probability, there is a $25 \%$ chance of identifying any single solution correctly. Given the limited information we were provided, the probability of identifying all four correctly is $0.4 \%$. Therefore, this level of accuracy is truly based on the sensor's performance.

Table 1. Comparison of predicted and actual (known) solutions.

\begin{tabular}{|c|c|c|c|c|c|c|c|c|}
\hline Solution & Buffer & BSA & SA & SA-PS Bead & Cat & Dog & Cow & Pig \\
\hline Prediction & & & & & Buffer & SA & SA-PS bead & SA-PS bead \\
\hline Actual & & & & & Buffer & SA & SA-PS bead & SA-PS bead \\
\hline $\begin{array}{c}\text { General } \\
\text { observations } \\
\text { and/or } \\
\text { comments } \\
\text { which led to } \\
\text { this conclusion }\end{array}$ & $\begin{array}{l}\text { No shifts } \\
\text { over noise } \\
\text { level; no } \\
\text { change in } \\
\text { cavity Q }\end{array}$ & $\begin{array}{c}\delta \lambda \sim 0.01 \mathrm{pm} ; \\
\text { minimal } \\
\text { change in } \mathrm{Q} ; \\
\text { minimal } \\
\text { change in } \\
\text { coupled power }\end{array}$ & $\begin{array}{c}\delta \lambda \sim 0.01 \mathrm{pm} ; \\
\text { minimal } \\
\text { change in } \mathrm{Q} ; \\
\text { minimal } \\
\text { change in } \\
\text { coupled } \\
\text { power }\end{array}$ & $\begin{array}{c}\delta \lambda>0.2 \mathrm{pm} \\
\text { and smaller } \\
\text { shifts; power } \\
\text { and Q } \\
\text { decrease; } \\
\text { scattering } \\
\text { observed }\end{array}$ & $\begin{array}{l}\text { No shifts } \\
\text { over noise } \\
\text { level; no } \\
\text { change in } \\
\text { cavity Q }\end{array}$ & $\begin{array}{c}\delta \lambda \\
\sim 0.01 \mathrm{pm}\end{array}$ & $\begin{array}{c}\delta \lambda>0.2 \mathrm{pm} \\
\text { and smaller } \\
\text { shifts; power } \\
\text { and Q decrease } \\
\text { over numerous } \\
\text { trials }\end{array}$ & $\begin{array}{c}\delta \lambda>0.2 \mathrm{pm} \\
\text { and smaller } \\
\text { shifts; power } \\
\text { and Q decrease } \\
\text { over numerous } \\
\text { trials }\end{array}$ \\
\hline
\end{tabular}

\subsection{Circulating Power and Thermal Effects}

As was mentioned in the procedures, the present series of experiments used very high input powers. This approach will give rise to very high circulating power or circulating intensity inside the optical cavity. Specifically, the circulating power can be analytically calculated as:

$$
P_{\text {circ }}=\frac{\lambda Q}{\pi^{2} n_{\text {eff }} R_{\text {meff }}} \frac{K}{(1+K)^{2}}
$$

where $\lambda$ is the resonant wavelength, $\mathrm{n}_{\mathrm{eff}}$ is the effective refractive index, $\mathrm{Q}$ is the intrinsic cavity quality factor, $\mathrm{K}$ is the coupling constant and $\mathrm{R}_{\text {meff }}$ is the effective radius of the mode. $\mathrm{R}_{\text {meff }}$ is related to the cavity major and minor diameters. Due to material absorption, temperature gradients are created [61]. Therefore, this circulating power can be observed as a change in the resonant wavelength according to:

$$
\Delta \lambda=\left(\frac{\lambda}{n_{\text {eff }}}\right)\left(\frac{d n}{d T}\right)(\Delta T)+\lambda \varepsilon \Delta T
$$

where $\mathrm{dn} / \mathrm{dT}$ is the effective thermo-optic coefficient, $\Delta \mathrm{T}$ is the temperature change, and $\varepsilon$ is the thermal expansion of the material. Typically, in dielectric systems, it is assumed that the index change dominates this equation. However, in certain situations, it has been shown that it is possible to remove the index-generated effect by balancing the positive $\mathrm{dn} / \mathrm{dT}$ of silica with a negative $\mathrm{dn} / \mathrm{dT}$ material, creating a net-zero dn/dT [62-66]. In sensing applications, this null shift is particularly desirable, as it will reduce the background signal. As can be seen throughout all of the noise measurements, we strived to achieve this neutrality condition by manipulating the optical field overlap between the silica 
device (positive $\mathrm{dn} / \mathrm{dT}$ ) and water (negative $\mathrm{dn} / \mathrm{dT}$ ). Due to subtle variations in device geometry, we observed a range of behaviors, including blue and red shifts as well as the ideal neutrality condition. However, the magnitude of these shifts is minor when compared to the shifts that would be observed if the device was operating in air, which has a positive $\mathrm{dn} / \mathrm{dT}$.

\section{Conclusions/Outlook}

In the present work, we have successfully performed a double-blind experiment verifying the ability of optical resonant cavities to detect and to identify single molecules based on resonant wavelength shifts. Additionally, we developed several new data analysis methods, enabling sub-linewidth detection limits without employing complex experimental techniques, such as heterodyning [42,67] or back-scatter [6]. These measurements have already inspired numerous subsequent research efforts into signal optimization and noise analysis as well as surface chemistry engineering [54,58,59]. Therefore, in addition to impacting the immediate field of resonant cavity biosensing, these findings have already and will continue to advance the broader field of biological and chemical sensing.

\section{Acknowledgments}

The authors would like to thank Heather Hunt (University of Missouri), Simin Mehrabani, and Rasheeda Hawk (University of Southern California) for surface functionalization of the devices, Matthew Reddick for the development of the LabView program used to remove taper jitter, and Hong Seok Choi and Xiaomin Zhang for the fabrication of devices. The authors thank Richard Flagan and Jason Gamba (California Institute of Technology), and Ashley Gamba (independent observer) for their assistance in the double-blind experiment; namely, making the initial solutions (J.G.), renaming them (A.G.), and holding the sealed envelope (R.F.) as well as aiding in the floating experiment design (R.F). This work was supported by the National Science Foundation (DBI-0852581 and ENG-1028440).

\section{Author Contributions}

A.A., C.S. and M.C. conceived and designed the experiments; C.S. and M.C. prepared solutions; C.S. and M.C. performed the experiments; A.A.C.S. and M.C. analyzed the data; A.A.C.S. and M.C. wrote the paper.

\section{Conflicts of Interest}

The authors declare no conflict of interest.

\section{References}

1. Luchansky, M.S.; Bailey, R.C. High-Q optical sensors for chemical and biological analysis. Anal. Chem. 2012, 84, 793-821.

2. Hunt, H.K.; Armani, A.M. Label-free biological and chemical sensors. Nanoscale 2010, 2, $1544-1559$. 
3. Erickson, D.; Mandal, S.; Yang, A.H.J.; Cordovez, B. Nanobiosensors: Optofluidic, electrical and mechanical approaches to biomolecular detection at the nanoscale. Microfluid Nanofluid 2008, 4, $33-52$.

4. Baaske, M.D.; Foreman, M.R.; Vollmer, F. Single-molecule nucleic acid interactions monitored on a label-free microcavity biosensor platform. Nat. Nanotech. 2014, 9, 933-939.

5. Shao, L.B.; Jiang, X.F.; Yu, X.C.; Li, B.B.; Clements, W.R.; Vollmer, F.; Wang, W.; Xiao, Y.F.; Gong, Q.H. Detection of single nanoparticles and lentiviruses using microcavity resonance broadening. Adv. Mater. 2013, 25, 5616-5620.

6. Knittel, J.; Swaim, J.D.; McAuslan, D.L.; Brawley, G.A.; Bowen, W.P. Back-scatter based whispering gallery mode sensing. Sci. Rep. 2013, 3, 2974.

7. Hawk, R.M.; Chistiakova, M.V.; Armani, A.M. Monitoring DNA hybridization using optical microcavities. Opt. Lett. 2013, 38, 4690-4693.

8. Dantham, V.R.; Holler, S.; Barbre, C.; Keng, D.; Kolchenko, V.; Arnold, S. Label-free detection of single protein using a nanoplasmonic-photonic hybrid microcavity. Nano Lett. 2013, 13, 3347-3351.

9. Wilson, K.A.; Finch, C.A.; Anderson, P.; Vollmer, F.; Hickman, J.J. Whispering gallery mode biosensor quantification of fibronectin adsorption kinetics onto alkylsilane monolayers and interpretation of resultant cellular response. Biomaterials 2012, 33, 225-236.

10. He, L.N.; Ozdemir, K.; Zhu, J.G.; Kim, W.; Yang, L. Detecting single viruses and nanoparticles using whispering gallery microlasers. Nat. Nanotechnol. 2011, 6, 428-432.

11. Zhu, J.; Ozdemir, S.K.; Xiao, Y.-F.; Li, L.; He, L.; Chen, D.-R.; Yang, L. On-chip single nanoparticle detection and sizing by mode splitting in an ultrahigh-q microresonator. Nat. Photon. 2009, 4, 46-49.

12. Robinson, J.T.; Chen, L.; Lipson, M. On-chip gas detection in silicon optical microcavities. Opt. Express 2008, 16, 4296-4301.

13. Ilchenko, V.S.; Maleki, L. Novel whispering-gallery resonators for lasers, modulators, and sensors. In Laser Resonators IV; Kudryashov, A.V., Paxton, A.H., Eds.; SPIE: Bellingham, WA, USA, 2001; Volume 4270, pp. 120-130.

14. Avino, S.; Krause, A.; Zullo, R.; Giorgini, A.; Malara, P.; de Natale, P.; Loock, H.P.; Gagliardi, G. Direct sensing in liquids using whispering-gallery-mode droplet resonators. Adv. Opt. Mater. 2014, 2, 1155-1159.

15. Cubillas, A.M.; Unterkofler, S.; Euser, T.G.; Etzold, B.J.; Jones, A.C.; Sadler, P.J.; Wasserscheid, P.; Russell, P.S.J. Photonic crystal fibres for chemical sensing and photochemistry. Chem. Soc. Rev. 2013, 42, 8629-8648.

16. Delezoide, C.; Ledoux-Rak, I.; Nguyen, C.T. General approach for the sensitivity analysis and optimization of integrated optical evanescent-wave sensors. JOSA B 2014, 31, 851-859.

17. Guo, Y.; Li, H.; Reddy, K.; Shelar, H.S.; Nittoor, V.R.; Fan, X. Optofluidic fabry-pérot cavity biosensor with integrated flow-through micro-/nanochannels. Appl. Phys. Lett. 2011, 98, 041104.

18. Kim, K.H.; Bahl, G.; Lee, W.; Liu, J.; Tomes, M.; Fan, X.; Carmon, T. Cavity optomechanics on a microfluidic resonator with water and viscous liquids. Light Sci. Appl. 2013, 2, e110.

19. Kukura, P.; Celebrano, M.; Renn, A.; Sandoghdar, V. Single-molecule sensitivity in optical absorption at room temperature. J. Phys. Chem. Lett. 2010, 1, 3323-3327. 
20. Langbein, W.; Chantada, L.; Nikolaev, N.I.; Ivanov, A.; Borri, P. Modelling the response of whispering-gallery-mode optical resonators for biosensing applications. In Proceedings of the 11th International Conference on Transparent Optical Networks (ICTON 2009), Azores, Portugal, 28 June-2 July 2009.

21. Lin, S.; Crozier, K.B. Planar silicon microrings as wavelength-multiplexed optical traps for storing and sensing particles. Lab Chip 2011, 11, 4047-4051.

22. Loock, H.-P.; Wentzell, P.D. Detection limits of chemical sensors: Applications and misapplications. Sens. Actuators B Chem. 2012, 173, 157-163.

23. Martinez-Cisneros, C.S.; Sanchez, S.; Xi, W.; Schmidt, O.G. Ultracompact three-dimensional tubular conductivity microsensors for ionic and biosensing applications. Nano Lett. 2014, 14, 2219-2224.

24. Morrissey, M.J.; Deasy, K.; Frawley, M.; Kumar, R.; Prel, E.; Russell, L.; Truong, V.G.; Nic Chormaic, S. Spectroscopy, manipulation and trapping of neutral atoms, molecules, and other particles using optical nanofibers: A review. Sensors 2013, 13, 10449-10481.

25. Piliarik, M.; Sandoghdar, V. Direct optical sensing of single unlabelled proteins and super-resolution imaging of their binding sites. Nat. Commun. 2014, 5, 4495.

26. Posani, K.; Tripathi, V.; Annamalai, S.; Weisse-Bernstein, N.; Krishna, S.; Perahia, R.; Crisafulli, O.; Painter, O. Nanoscale quantum dot infrared sensors with photonic crystal cavity. Appl. Phys. Lett. 2006, 88, 151104.

27. Ryckeboer, E.; Bockstaele, R.; Vanslembrouck, M.; Baets, R. Glucose sensing by waveguide-based absorption spectroscopy on a silicon chip. Biomed. Opt. Express 2014, 5, 1636-1648.

28. Smolka, S.; Barth, M.; Benson, O. Selectively coated photonic crystal fiber for highly sensitive fluorescence detection. Appl. Phys. Lett. 2007, 90, 111101.

29. Soteropulos, C.E.; Zurick, K.M.; Bernards, M.T.; Hunt, H.K. Tailoring the protein adsorption properties of whispering gallery mode optical biosensors. Langmuir 2012, 28, 15743-15750.

30. Stiebeiner, A.; Rehband, O.; Garcia-Fernandez, R.; Rauschenbeutel, A. Ultra-sensitive fluorescence spectroscopy of isolated surface-adsorbed molecules using an optical nanofiber. Opt. Express 2009, 17, 21704-21711.

31. Van Eeghem, A.; Werquin, S.; Vanderleyden, E.; Bienstman, P.; Dubruel, P. Functionalization of nanophotonic silicon-on-insulator biosensor chips for real-time DNA detection. In Proceedings of the European Conference on Novel Technologies for in vitro Diagnostics (DIATECH 2014), Leuven, Belgium, 6-8 October 2014; pp. 34-35.

32. Ward, J.; Benson, O. Wgm microresonators: Sensing, lasing and fundamental optics with microspheres. Laser Photon. Rev. 2011, 5, 553-570.

33. Ward, J.M.; Dhasmana, N.; Chormaic, S.N. Hollow core, whispering gallery resonator sensors. Eur. Phys. J. Spec. Top. 2014, 223, 1917-1935.

34. Wiersig, J. Enhancing the sensitivity of frequency and energy splitting detection by using exceptional points: Application to microcavity sensors for single-particle detection. Phys. Rev. Lett. 2014, 112, 203901.

35. Yang, D.; Kita, S.; Liang, F.; Wang, C.; Tian, H.; Ji, Y.; Lončar, M.; Quan, Q. High sensitivity and high Q-factor nanoslotted parallel quadrabeam photonic crystal cavity for real-time and label-free sensing. Appl. Phys. Lett. 2014, 105, 063118. 
36. Hawk, R.; Armani, A.M. Label-free detection of 5 'hydroxymethylcytosine within cpg islands using optical sensors. Biosens. Bioelectron. 2015, 65, 198-203.

37. Socorro, A.B.; Soltani, S.; Villar, I.D.; Corres, J.M.; Armani, A.M. Temperature sensor based on a hybrid ito-silica resonant cavity. Opt. Express 2015, 23, 1930-1937.

38. Aardahl, C.L.; Foss, W.R.; Davis, E.J. The effects of optical resonances on raman analysis of liquid aerosols. J. Aerosol Sci. 1996, 27, 1015-1033.

39. Eversole, J.D.; Lin, H.B.; Merritt, C.D.; Campillo, A.J. Absorption-spectroscopy using microdroplet resonance fluorescence intensities. Appl. Spectrosc. 1994, 48, 373-381.

40. Rosenberger, A.T.; Rezac, J.P. Evanescent-wave sensor using microsphere whispering-gallery modes. In Laser Resonators III; Kudryashov, A.V., Paxton, A.H., Eds.; SPIE: Bellingham, WA, USA, 2000; Volume 3930, pp. 186-192.

41. Yang, J.; Guo, L.J. Optical sensors based on active microcavities. IEEE J. Sel. Top. Quantum Electron. 2006, 12, 143-147.

42. Maker, A.J.; Armani, A.M. Heterodyned toroidal microlaser sensor. Appl. Phys. Lett. 2013, 103, 123302.

43. Mehrabani, S.; Kwong, P.; Gupta, M.; Armani, A.M. Hybrid microcavity humidity sensor. Appl. Phys. Lett. 2013, 102, 241101.

44. Cheema, M.I.; Mehrabani, S.; Peter, Y.-A.; Armani, A.M.; Kirk, A.G. Simultaneous measurement of quality factor and wavelength shift by phase shift microcavity ring down spectroscopy. Opt. Express 2012, 20, 9090-9098.

45. Matsko, A.B.; Ilchenko, V.S. Optical resonators with whispering-gallery modes-part I: Basics. IEEE J. Sel. Top. Quantum Electron. 2006, 12, 3-14.

46. Pitt, B.; Zannad, F.; Remme, W.J.; Cody, R.; Castaigne, A.; Perez, A.; Palensky, J.; Wittes, J. The effect of spironolactone on morbidity and mortality in patients with severe heart failure. N. Engl. J. Med. 1999, 341, 709-717.

47. Sano, M.; Ernesto, C.; Thomas, R.G.; Klauber, M.R.; Schafer, K.; Grundman, M.; Woodbury, P.; Growdon, J.; Cotman, D.W.; Pfeiffer, E.; et al. A controlled trial of selegiline, alpha-tocopherol, or both as treatment for alzheimer's disease. N. Engl. J. Med. 1997, 336, 1216-1222.

48. Armani, D.K.; Kippenberg, T.J.; Spillane, S.M.; Vahala, K.J. Ultra-high-q toroid microcavity on a chip. Nature 2003, 421, 925-928.

49. Maker, A.J.; Armani, A.M. Fabrication of silica ultra high quality factor microresonators. J. Vis. Exp. 2012, doi:10.3791/4164.

50. Armani, A.M.; Armani, D.K.; Min, B.; Vahala, K.J.; Spillane, S.M. Ultra-high-Q microcavity operation in $\mathrm{H}_{2} \mathrm{O}$ and $\mathrm{D}_{2} \mathrm{O}$. Appl. Phys. Lett. 2005, 87, 151118.

51. Vernooy, D.W.; Ilchenko, V.S.; Mabuchi, H.; Streed, E.W.; Kimble, H.J. High-Q measurements of fused-silica microspheres in the near infrared. Opt. Lett. 1998, 23, 247-249.

52. Gorodetsky, M.L.; Savchenkov, A.A.; Ilchenko, V.S. Ultimate Q of optical microsphere resonators. Opt. Lett. 1996, 21, 453-455.

53. Hunt, H.K.; Soteropulos, C.; Armani, A.M. Bioconjugation strategies for microtoroidal optical resonator. Sensors 2010, 10, 9317-9336.

54. Hunt, H.K.; Armani, A.M. Bioconjugation strategies for label-free optical microcavity sensors. IEEE J. Sel. Top. Quantum Electron. 2014, 20, 6900213. 
55. Yariv, A. Universal relations for coupling of optical power between microresonators and dielectric waveguides. Electron. Lett. 2000, 36, 321-333.

56. Chistiakova, M.; Armani, A.M. Cascaded raman microlaser in air and buffer. Opt. Lett. 2012, 37, 4068-4070.

57. Gamba, J.M.; Flagan, R.C. Flow-enhanced transient response in whispering gallery mode biosensors. Appl. Phys. Lett. 2011, 99, 253705.

58. Cheema, M.I.; Shi, C.; Armani, A.M.; Kirk, A.G. Optimizing the signal to noise ratio of microcavity sensors. IEEE Photon. Technol. Lett. 2014, 26, 2023-2026.

59. Cheema, M.I.; Khan, U.A.; Armani, A.M.; Kirk, A.G. Towards more accurate microcavity sensors: Maximum likelihood estimation applied to a combination of quality factor and wavelength shifts. Opt. Express 2013, 21, 22817-22828.

60. Shi, C.; Mehrabani, S.; Armani, A.M. Leveraging bimodal kinetics to improve detection specificity. Opt. Lett. 2012, 37, 1643-1645.

61. Soltani, S.; Armani, A.M. Optothermal transport behavior in whispering gallery mode optical cavities. Appl. Phys. Lett. 2014, 105, 051111.

62. Le, T.; Savchenkov, A.; Yu, N.; Maleki, L.; WH, S. Optical resonant sensors: A method to reduce the effect of thermal drift. Appl. Opt. 2009, 48, 458-463.

63. Choi, H.-S.; Armani, A.M. Thermal non-linear effects in hybrid optical microresonators. Appl. Phys. Lett. 2010, 97, 223306.

64. He, L.; Xiao, Y.F.; Dong, C.; Zhu, J.; Gaddam, V.; Yang, L. Compensation of thermal refraction effect in high-Q toroidal microresonator by polydimethylsiloxane coating. Appl. Phys. Lett. 2008, 93, 201102.

65. Rokhsari, H.; Spillane, S.M.; Vahala, K.J. Loss characterization in microcavities using the thermal bistability effect. Appl. Phys. Lett. 2004, 85, 3029-3031.

66. Choi, H.S.; Ismail, S.; Armani, A.M. Studying polymer thin films with hybrid optical microcavities. Opt. Lett. 2011, 36, 2152-2154.

67. Lu, T.; Lee, H.; Chen, T.; Herchak, S.; Kim, J.-H.; Fraser, S.E.; Flagan, R.C.; Vahala, K. High sensitivity nanoparticle detection using optical microcavities. Proc. Natl. Acad. Sci. USA 2011, 108, 5976-5979.

(C) 2015 by the authors; licensee MDPI, Basel, Switzerland. This article is an open access article distributed under the terms and conditions of the Creative Commons Attribution license (http://creativecommons.org/licenses/by/4.0/). 